\title{
NonDex: A Tool for Detecting and Debugging Wrong Assumptions on Java API Specifications
}

\author{
Alex Gyori, Ben Lambeth, August Shi, Owolabi Legunsen, and Darko Marinov \\ Department of Computer Science, University of Illinois at Urbana-Champaign \\ Urbana, IL 61801, USA \\ \{gyori, blambet2, awshi2, legunse2, marinov\}@illinois.edu
}

\begin{abstract}
We present NonDEx, a tool for detecting and debugging wrong assumptions on Java APIs. Some APIs have underdetermined specifications to allow implementations to achieve different goals, e.g., to optimize performance. When clients of such APIs assume stronger-than-specified guarantees, the resulting client code can fail. For example, HashSet's iteration order is underdetermined, and code assuming some implementation-specific iteration order can fail. NONDEX helps to proactively detect and debug such wrong assumptions. NONDEx performs detection by randomly exploring different behaviors of underdetermined APIs during test execution. When a test fails during exploration, NonDEx searches for the invocation instance of the API that caused the failure. NONDEx is open source, well-integrated with Maven, and also runs from the command line. During our experiments with the NonDex Maven plugin, we detected 21 new bugs in eight Java projects from GitHub, and, using the debugging feature of NonDex, we identified the underlying wrong assumptions for these 21 new bugs and 54 previously detected bugs. We opened 13 pull requests; developers already accepted 12 , and one project changed the continuous-integration configuration to run NoNDEX on every push. The demo video is at: https://youtu.be/h3a9ONkC59c
\end{abstract}

\section{CCS Concepts}

-Software defect analysis $\rightarrow$ Software testing and debugging;

\section{Keywords}

NonDex, flaky tests, underdetermined API

\section{INTRODUCTION}

Some commonly used Java APIs have underdetermined specifications. Following Liskov 9, we say that a specification is underdetermined if it allows multiple implementations to return different results for the same input, even

Permission to make digital or hard copies of all or part of this work for personal or classroom use is granted without fee provided that copies are not made or distributed for profit or commercial advantage and that copies bear this notice and the full citation on the first page. Copyrights for components of this work owned by others than ACM must be honored. Abstracting with credit is permitted. To copy otherwise, or republish, to post on servers or to redistribute to lists, requires prior specific permission and/or a fee. Request permissions from Permissions@acm.org.

FSE' 16, November 13-18, 2016, Seattle, WA, USA

(c) 2016 ACM. 978-1-4503-4218-6/16/11 ..\$15.00

http://dx.doi.org/10.1145/2950290.2983932 if each implementation is itself deterministic and always returns the same result for the same input. We refer to an API with such a specification as an underdetermined API. An example underdetermined API is the iterator() method in java.util.HashSet, whose Javadoc specification states, "The elements are returned in no particular order" [4]. Similarly, libraries for generating JSON typically do not guarantee any order for elements in a JSON document 7]. Having such underdetermined specifications is good because it gives implementers of the underdetermined APIs the flexibility to optimize various implementations of the API for different goals, e.g., they may optimize performance in different ways. However, it is important to precisely state underdetermined specifications in the API documentation to express all expected behaviors of all API's implementations.

Unfortunately, even when underdetermined APIs have precise documentation, developers do make wrong assumptions about the underdetermined APIs. While such APIs could allow even non-deterministic implementations, each typical implementation is deterministic, i.e., two runs of the same implementation (on the same platform) give the same result for the same input. For example, two runs of a program that iterates over a Hashset may return the elements in the same order. However, such deterministic implementations can mislead the developers of API clients, who may assume that all API implementations are guaranteed to behave in the same deterministic manner. For HashSet, while one Java version could provide a deterministic iteration order, different Java versions provide different iteration orders (e.g., the order in Java 7 differs from the order in Java 8). If clients of an underdetermined API assume stronger-than-specified guarantees, the resulting code can fail when the API implementation changes, albeit the specification remains the same. A well-known example of such wrong assumptions is that many projects with JUnit tests relied on a particular order in which tests are executed; when these projects upgraded from Java 6 to Java 7, many tests failed because the order changed from Java 6 to Java 7 8], albeit the specification of the order did not change.

The state-of-the-practice in detecting the negative effects of wrong assumptions on underdetermined APIs is rather reactive. Most developers discover such assumptions only after failures happen (e.g., after platforms are changed). Unexpected behaviors can also manifest as so called "flaky" tests [10], which can pass or fail seemingly without any changes to the code. A flaky test that assumes a certain behavior, which is not guaranteed by the API specification, can fail when the API implementation changes. The devel- 
opers of several projects followed a reactive practice in the past by fixing their own code as a result of test failures due to wrong assumptions $3,6,8,11,12,17$.

We propose NonDex, a tool to proactively detect wrong assumptions on underdetermined APIs by randomly exploring different allowed behaviors during test execution. For HashSet, for example, with its underdetermined iteration order, NonDEx randomly explores different iteration orders, which can proactively detect failures of tests that iterate over HashSet, either directly in the test code itself or in the code under test. Once a test fails during exploration, that failure demonstrates that the code makes some wrong assumption. NonDex also helps in debugging by pinpointing the location where the assumption is being made; the debugging feature searches for the dynamic invocation of the API whose exploration causes the failure. NonDEx can be run as a stand-alone command-line tool and can thus be run on any Java-based project, regardless of the build system used. We additionally provide a NonDEx Maven plugin that allows easy integration with projects that use the Maven build system. Developers can run NonDex, e.g., during continuous integration, to check for wrong assumptions on Java APIs. It is often more cost-effective to proactively detect bugs right when they are introduced rather than reactively, after they manifest.

This paper makes several contributions to the tool design and implementation. We recently published the research idea of NonDex [16] and evaluated it using a prototype implementation which modified the OpenJDK JVM and was not easily portable. The current NonDEx tool improves four aspects: (1) the tool is pure Java and is widely portable due to a novel instrumentation mechanism, (2) the Maven plugin is new and allowed us to scale our experiments, (3) the debugging feature is also new, and (4) NonDEx is now open source on GitHub and is under active development 15]. While experimenting with the new implementation of NONDEx, we detected 21 new bugs in eight Maven-based Java projects from GitHub. Using the new debugging feature, we identified the underlying wrong assumptions for these 21 new bugs and 54 previously detected bugs. We opened 13 pull requests, and developers already accepted 12. Further, the Checkstyle project, in which we found 5 bugs, integrated NonDEX into their continuous-integration configuration to run on every push 2]. While trying out NonDEx at Google, we learned that Google had previously implemented a similar exploration in March 2015 ${ }^{1}$ confirming that the problem addressed by NoNDEX is important in practice.

\section{USAGE}

We describe how to use NonDex as a Maven plugin 13 or from command line.

\subsection{Integration with Maven}

NonDex is most easily integrated in the testing process via the Maven plugin, available from the Maven Central repository 14. To use NonDEx, developers only need to add NonDex as a plugin entry to their build file (pom.xml).

\footnotetext{
${ }^{1}$ The Google implementation predates our earlier publication [16], but their implementation handles only one API, has only one mode, does not integrate with Maven, and is not open source, while NonDEx handles 41 APIs, has two modes, integrates with Maven, and is open source.
}

The NonDex Maven plugin provides three goals: nondex:nondex runs all tests while exploring different random behaviors of 41 underdetermined APIs from the standard Java library and reports failing tests; nondex:debug reruns test(s) that failed during exploration to locate the invocation(s) of an underdetermined API that made the wrong assumption; and nondex:clean deletes the .nondex directories where NonDEx stores auxiliary files during exploration and debugging. The NonDex Maven plugin also takes several optional arguments (with sensible defaults): nondexSeed is the main seed to use for randomization; nondexRuns is the number of runs to perform, each with a different starting seed computed from the main seed; and nondexMode is one of two exploration modes, ONE and FULL, where ONE has more determinism than FULL as described in our prior work 16 .

\subsection{Command-Line Invocation}

NonDex can be run from command line. First, the instrumentation engine should be run once to (i) modify the code for underdetermined APIs by adding the random exploration code and (ii) package the modified code as a JAR file, say, nondex-rt.jar. Next, the user can run NonDEx on any Java application by adding to the bootclasspath (not the regular classpath) the generated nondex-rt.jar and the nondex-common.jar which contains code for configuring NoNDEX and logging its output. The two JAR files are added to the bootclasspath because nondex-rt. jar modifies the behavior of several classes in the standard library, and those classes must be loaded from the instrumented JAR. The following example commands will run a class Main using NoNDEx to randomly explore different allowed behaviors of the underdetermined APIs:

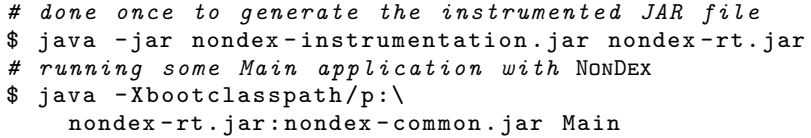

From command line, the user can optionally provide a random seed and a mode (by default FULL) for NoNDEX.

\section{TECHNIQUE AND IMPLEMENTATION}

NonDex has two user-facing phases: (i) detection finds tests that pass without NonDex but fail when NonDex explores different allowed behaviors - such failures indicate wrong assumption(s) made on underdetermined APIs; and (ii) debugging searches through detected failures to find the underdetermined APIs on which wrong assumptions were made and to identify the invocation(s) making such assumptions. Currently, NonDex exploration handles 41 underdetermined APIs that we manually identified from the following packages java.lang, java.util, java.io, and java.text; we used 30 of these underdetermined APIs in our earlier paper 16 . Table I $]^{2}$.

Internally, NoNDEx consists of four components: (1) the instrumentation engine modifies the API classes in the standard library to add code for random exploration, (2) the runner executes the program on the instrumented library, (3) the detector reruns the program a specified number of times to randomly explore different behaviors, and (4) the

\footnotetext{
${ }^{2}$ The publicly released NONDEx does not handle the native hashCode, because it did not expose any bugs during our experiments and would unnecessarily complicate the tool.
} 


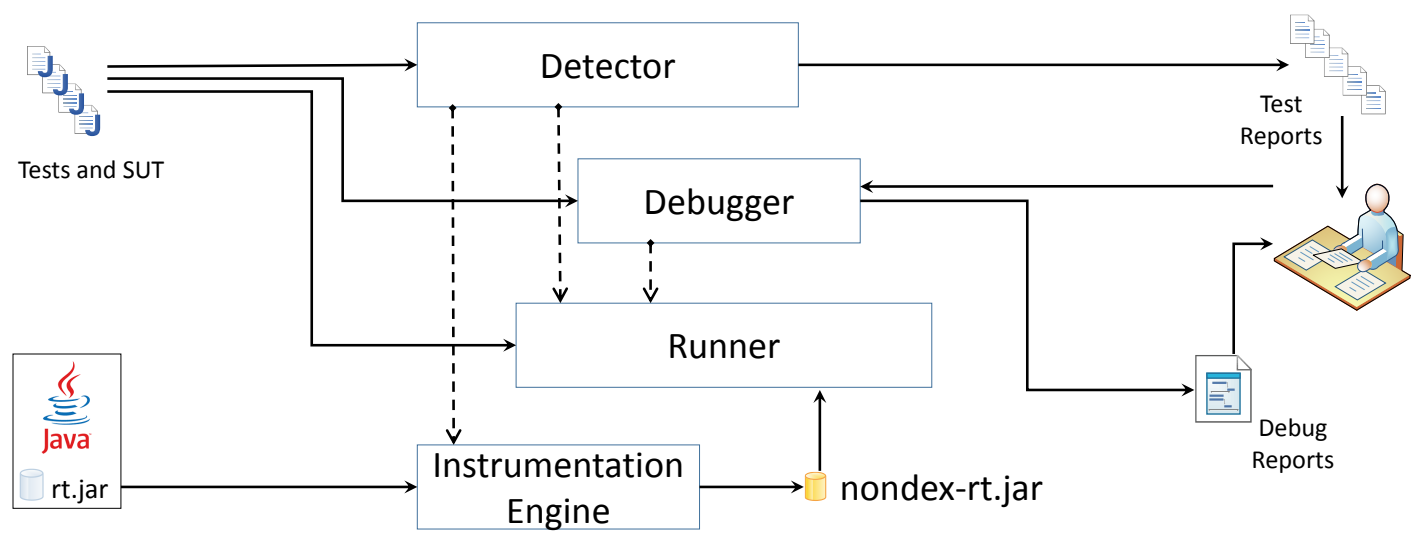

Figure 1: High-level architecture of the key NonDex components; see Section 3 for the description

debugger identifies the API invocation(s) where a wrong assumption was made. Figure 1 shows an architectural overview of the NonDEx components, and the following subsections describe each component.

\subsection{Instrumentation Engine}

The goal of the instrumentation engine is to modify standard Java library classes to allow random exploration. The challenge is to develop instrumentation that can automatically handle a large number of Java versions. For our original prototype [16], we manually modified the Java sources of the relevant files, compiled them, and used them in place of the original files. However, this solution was brittle, because the tool would often not work unless the exact same Java version (e.g., 1.8.0-b132) was used for the run as the version for which we manually modified the sources. The reason the tool did not work was that some internal parts of the modified files changed between Java versions, even when the signatures of the public APIs we modified did not change. Hence, we developed our current, instrumentation-based solution that is much more robust, and we have tested it on 14 different versions of OpenJDK and Oracle's JDK implementations of Java 8, on Linux, OS X, and Windows.

The instrumentation engine takes as input the rt.jar file containing the classfiles of the standard Java library that will be used when running the tests. The instrumentation engine selects from rt.jar the classfiles corresponding to the APIs that should be modified to add random exploration. For APIs that should be modified and return an array, our instrumentation simply adds a custom NONDEX helper method to explore different orders of the returned array (effectively randomly permuting the array before returning it). This modification is robust as long as the type signature of the API does not change. The instrumentation is much more involved for the (Concurrent) HashMap classes, because their iterators are lazy, implemented as private data structures that change even within the same Java major version, e.g., the HashMap iterator was implemented using a class called Entry until OpenJDK version 1.8.0-b108 [5] and using a class called Node since then; we developed customized instrumenters that can generate appropriate modified code based on whether rt.jar uses Entry or Node. This modification would need to change in the future if the standard Java library implements HashMap using a third approach. We used ASM 1] to implement all classfile manipulation.
Performing instrumentation from scratch on every run is unnecessary, so we reuse each previously instrumented class in subsequent runs, as long as the instrumented class from rt.jar did not change. (The original class does not change until/unless the user switches to another version of Java.) To decide when to reuse the instrumented classes, NonDex stores for each instrumented class the checksum of the classfile from the rt.jar that was instrumented.

\subsection{Runner}

The runner is a thin layer of code that enables random execution for APIs instrumented by NonDEx. On every invocation of an instrumented API, the runner randomly chooses one behavior from the behaviors appropriate for that API. NonDEx currently supports two kinds of behaviors: (1) permutation for APIs where order is underdetermined, and (2) extensions for APIs where only lower bounds on array size(s) are specified. The runner takes as inputs (i) a random seed, which completely determines the choice of behaviors, (ii) the mode of exploration - ONE or FULL (the two modes differ in the kind of wrong assumptions they can detect, as described in detail in our prior work 16$]^{3}$, and (iii) optionally the range of choices to be randomized (which is used by debugging).

\subsection{Detector}

The detector first runs all tests once without randomization and then calls the NoNDEx runner a number of times, with different random seeds, to rerun all the tests. The detector reports tests that pass without NONDEX randomization but fail with NONDEX randomization; such tests likely ${ }^{4}$ make wrong assumptions on underdetermined APIs. The detector first runs the tests without NONDEX because tests that fail on their own are due to some other causes and should not be reported as failures due to wrong assumptions. After the first run, the detector invokes the instrumentation engine to create the instrumented APIs before it starts running tests with NoNDEX.

\footnotetext{
${ }^{3}$ We originally evaluated four different modes, but the publicly released NONDEX offers only two modes, ONE and FULL, because they are the easiest to understand and correspond to the two extremes of non-determinism.

${ }^{4}$ The tests may be flaky 10 due to other reasons and fail irrespective of NONDEX.
} 
The detector stores information about failing tests in a .nondex directory which also contains information about each execution, without and with NONDEx, as well as the configuration used for test executions, the seed needed to reproduce the failure and the number of invocations of the runner's choice generator; this number helps the debugging phase to search for the invocation(s) that caused the failure(s).

\subsection{Debugger}

When a test fails with NoNDEx, the test may invoke several underdetermined APIs, e.g., it may iterate over several HashSet objects. Many of these invocations are correct, making no wrong assumptions, so manually locating the invocation(s) that caused the detected failure can be tedious. The debugging phase automatically identifies such invocation(s).

To locate such invocation(s), NonDEx uses a binary search that keeps track of a range of API invocations and selectively enables exploration for half of them. Even for disabled invocations, our search advances the random-number generator, i.e., NonDEx still calls the random-number generator to shuffle the order of elements, but NonDEx returns the original, not the shuffled, order. (Without this control, the search could get different behaviors for the same random seed, making it harder to reproduce the failure.) Debugging continues until a single invocation is identified, or the remaining range cannot be further halved. If a single invocation cannot be identified from running just one test method, NonDEx re-starts debugging for the entire test class, and if again a single invocation cannot be identified, NonDEx re-starts debugging for the entire test suite. Debugging is repeated for each failing test reported by the detector.

The debugging phase reports to the user an API that causes the detected failure together with the call stack of the API's invocation which further helps in localizing the context in which the wrong assumption was made. In our prior work [16], we performed all debugging manually; after implementing automated debugging, we found that we had made an error in manually identifying the root cause of one failure, which shows that the automated debugging helps to more reliably identify the root causes.

\section{EXPERIMENTS}

Our initial NonDex prototype [16] detected dozens of tests in open-source code with wrong assumptions on underdetermined APIs. We experimented with our new NonDEX tool by adding it to pom.xml for several open-source projects, running nondex:nondex (which detected 21 new bugs), and running nondex: debug (for the 21 new bugs and 54 old bugs).

\subsection{Detecting Failures}

To test the NonDex tool in general and the NonDex Maven plugin in particular, we integrated NonDEx in the pom.xml files of several Maven-based projects from GitHub. Our goal was to test whether NonDex works with these projects "out-of-the-box" and not necessarily to detect any bugs. We found that integrating NonDEx into these projects was indeed easy, and that by just adding a few lines to pom.xml, we could run NoNDEX on all these projects. NONDEx worked well with projects that use different testing frameworks (e.g., JUnit 4, JUnit 3, and TestNG) and even various test runners (e.g., parameterized tests). Along the way, we also detected 21 new failing tests in eight projects (eight in alibaba/fastjason, five in checkstyle/checkstyle, three in nutzam/nutz, and one in each of alibaba/druid, bukkit/bukkit, jankotek/mapdb, pedrovgs.algorithms/algorithms, and perwendel/spark).

\subsection{Debugging Failures}

We further applied the automated NonDEx debugging on these 21 newly detected and 54 previously detected failing tests to determine the root cause of each failure. The number of underdetermined API invocations that NONDEX randomized per failure ranged from 5 to 9,710 . The results showed that our simple binary-search debugging works extremely well for these cases - for 74 out of 75 failures, NoNDEX minimized the cause down to only one invocation; the remaining failure is for a test written in JUnit 3 for which the Surefire Maven plugin (used by NoNDEx to run tests) cannot easily run single test methods. We also counted the number of wrong assumptions on various APIs supported by NoNDEx; the invocations causing the failures were getDeclaredFields (41 cases), HashMap iteration (32 cases), and getGenericExceptionTypes (1 case). Because binary search is simple, we were surprised that it sufficed to identify only one invocation in all but one of the cases we tried. In the future, we plan to explore more sophisticated search strategies, such as delta debugging 18, and automated fixing.

\subsection{Case Studies and Adoption}

We opened 13 pull requests (PRs) for failures detected by NonDex, reporting the issue and providing a fix, in four open-source projects: five in alibaba/fastjson, five in checkstyle/checkstyle, two in scribejava/scribejava, and one in square/retrofit. We did not open PRs for all bugs that NONDEx detected because we are not experts in the projects and could not easily provide a fix for each bug. All PRs we opened were accepted by developers except one PR in alibaba/fastjson. One of the developers of Checkstyle was quite pleased with the PRs we opened, asked us about the tool we used to detect the issues, and recommended that we integrate NonDEx in their continuous integration; we indeed integrated NONDEX in both pom.xml and .travis.yml for Checkstyle 2]. Furthermore, we are piloting the use of NonDex in a software testing course to educate students about wrong assumptions on underdetermined APIs. Students are using NonDex to find issues both in their own code and in open-source projects they are familiar with. Overall, we found NonDEx to be robust enough for use both in real-world projects and in teaching.

\section{CONCLUSIONS}

We presented the design and implementation of the NoNDEx tool we developed to help in detecting and debugging wrong assumptions on underdetermined APIs in Java. NONDEX is open source, integrates well with Maven, and can be also run from the command line. Using NonDex, we detected and debugged several failures in open-source projects.

\section{ACKNOWLEDGMENTS}

We thank Lamyaa Eloussi, Zach Gleason, Farah Hariri, John Micco, Timothy Tunnell, and Tifany Yung for providing feedback and suggesting improvements to the NONDEx tool. This research was partially supported by the NSF Grant Nos. CCF-1409423, CCF-1421503, and CCF-1439957. We also gratefully acknowledge the Google Faculty Award. 


\section{REFERENCES}

[1] ASM: A code manipulation tool to implement adaptable systems. In Adaptable and extensible component systems, Grenoble, France, Nov. 2002.

[2] Checkstyle pull request integrating NonDex. https://github.com/checkstyle/checkstyle/pull/3393

[3] Fix internal data ordering. https://github.com/ geosolutions-it/geoserver-manager/commit/5447c06.

[4] HashSet Javadoc. https://docs.oracle.com/javase/8/ docs/api/java/util/HashSet.html

[5] Improvements to HashMap/LinkedHashMap use of bins/buckets and trees (red/black), Sept. 2013. http:// hg.openjdk.java.net/jdk8/jdk8/jdk/rev/d62c911aebbb

[6] JUnit 4.11 - What's new? Test execution order. http://randomallsorts.blogspot.com/2012/12/ junit-411-whats-new-test-execution-order.html

[7] JSON. http://www.json.org/

[8] JUnit and Java 7. http://intellijava.blogspot.com/ 2012/05/junit-and-java-7.html.

[9] B. Liskov and J. Guttag. Program Development in Java: Abstraction, Specification, and Object-Oriented Design. Addison-Wesley, 2000.
[10] Q. Luo, F. Hariri, L. Eloussi, and D. Marinov. An empirical analysis of flaky tests. In FSE, 2014.

[11] Maintaining the order of JUnit3 tests with JDK 1.7. http://www.coderanch.com/t/600985/Testing/ Maintaining-order-JUnit-tests-JDK.

[12] all and sundry: JUnit test method ordering. http://www.java-allandsundry.com/2013/01/ junit-test-method-ordering.html

[13] Maven. https://maven.apache.org/.

[14] Maven Central. https://repo1.maven.org/maven2/ edu/illinois/nondex-maven-plugin/

[15] NonDex Source Code. https://github.com/TestingResearchIllinois/NonDex

[16] A. Shi, A. Gyori, O. Legunsen, and D. Marinov. Detecting assumptions on deterministic implementations of non-deterministic specifications. In ICST, 2016.

[17] Must Use LinkedHashMap and LinkedList... https://github.com/EsotericSoftware/yamlbeans/ commit/1517822

[18] A. Zeller and R. Hildebrandt. Simplifying and isolating failure-inducing input. TSE, 28(2), 2002. 